\title{
CONVERGENCE RATE \\ OF A MULTIPLICATIVE SCHWARZ \\ METHOD FOR STRONGLY NONLINEAR \\ VARIATIONAL INEQUALITIES
}

\author{
L. Badea \\ Institute of Mathematics, \\ Romanian Academy of Sciences, \\ P.O. Box 1-764, \\ RO-70700 Bucharest, Romania \\ Ibadea@imar.ro
}

Abstract We prove the convergence and estimate the error of a general algorithm for the minimization of non-quadratic functionals over a convex set in a reflexive Banach space, provided that the convex set verifies a certain assumption. In the case of the Sobolev spaces, our algorithm is exactly a variant of the Schwarz domain decomposition method, and we prove that the introduced assumption holds if the convex set is defined by constraints on the function values almost everywhere in the domain. In the end of the paper we give some numerical examples concerning the two-obstacle problem of a nonlinear elastic membrane.

Keywords: domain decomposition methods, Schwarz method, nonlinear variational inequalities, nonlinear minimization, obstacle problems

\section{Introduction}

The literature on the domain decomposition methods is very large and it is motivated by an increasing need on the solution of large-scale problems since these methods provide numerical solvers which are efficient and parallelizable on multi-processor machines. However, to our knowledge, very few papers deal with the application of these methods to nonlinear problems.

The main goal of this paper is to give an error estimate for a Schwarz domain decomposition method applied to the minimization of the non quadratic functionals over a convex set which is not supposed to be decomposed as a sum of subconvex sets. The convergence of a domain de-

The original version of this chapter was revised: The copyright line was incorrect. This has been corrected. The Erratum to this chapter is available at DOI: 10.1007/978-0-387-35690-7_44

V. Barbu et al. (eds.), Analysis and Optimization of Differential Systems

(C) IFIP International Federation for Information Processing 2003 
composition algorithm solving variational inequalities coming from the minimization of quadratic functionals over convex sets which are defined by constraints on the function values at the points of the domain is proved in [2]. In [9], it is proved that the multiplicative space decomposition method applied to the minimization without constraints of a differentiable and convex functional defined in a reflexive Banach space uniformly converges. In [3], it is proved that the method in [2] converges for the more general conditions given in [9] in the case of the quadratic functional minimization. We generalize in this paper the results in [3] and [9] to the minimization of the non quadratic functionals.

The paper is organized as follows. In Section 2, we state the multiplicative Schwarz method for nonlinear variational inequalities as a subspace correction method in a general reflexive Banach space for the minimization of non quadratic functionals, and we prove the convergence of this algorithm provided that a certain assumption holds. In Section 3, under a little stronger assumption, which essentially introduces a constant depending on the convex set and the space decomposition, we estimate the error of the algorithm. Section 4 is devoted to the convergence of the method in Sobolev spaces, proving that the introduced assumptions hold. For the Sobolev spaces, the algorithm is exactly a variant of the Schwarz method. Finally, in Section 5, we illustrate the method by numerical examples concerning the two-obstacle problem of a nonlinear elastic membrane.

\section{General convergence result}

Let $V$ be a reflexive Banach space and $V_{1}, \cdots, V_{m}$, be some closed subspaces of $V$. Also, we consider a non empty closed convex set $K \subset V$, and we make the following

Assumption 1. For any $w, v \in K$ and $w_{i} \in V_{i}$ with $w+\sum_{j=1}^{i} w_{j} \in K$,
$i=1, \cdots, m$, there exist $v_{i} \in V_{i}, i=1, \cdots, m$, satisfying

$$
\begin{gathered}
w+\sum_{j=1}^{i-1} w_{j}+v_{i} \in K \text { for } i=1, \cdots, m, \\
v-w=\sum_{i=1}^{m} v_{i}
\end{gathered}
$$

and the application from $V \times V_{1} \times \cdots \times V_{m}$ to $V_{1} \times \cdots \times V_{m}$

$$
\left(v-w, w_{1}, \cdots, w_{m}\right) \rightarrow\left(v_{1}, \cdots, v_{m}\right) .
$$

is bounded, i.e. it transforms the bounded sets in some bounded sets. 
This assumption looks to be complicated enough, but, as we shall see in Section 4, it holds for problems in which we use the Sobolev spaces and the convex set $K$ is defined by constraints of the function values at the points of the domain. We consider a Gâteaux differentiable functional $F: K \rightarrow R$, which will be assumed to be coercive if $K$ is not bounded. We assume that for any real number $M>0$, if we write $L_{M}=\quad \sup \quad\|v-u\|$, there exist two functions $\|v\|,\|u\| l e M, v, u \in K$

$\alpha_{M}, \beta_{M}:\left[0, L_{M}\right] \rightarrow \mathbf{R}^{+}$, such that

$\alpha_{M}$ is continuous and strictly increasing, and $\alpha_{M}(0)=0$,

$$
\beta_{M} \text { is continuous at } 0 \text { and } \beta_{M}(0)=0,
$$

and satisfying for any $u, v \in K$ with $\|u\|,\|v\| \leq M$,

$$
\begin{gathered}
<F^{\prime}(v)-F^{\prime}(u), v-u>\geq \alpha_{M}(\|v-u\|), \\
\beta_{M}(\|v-u\|) \geq\left\|F^{\prime}(v)-F^{\prime}(u)\right\|_{V^{\prime}},
\end{gathered}
$$

where $F^{\prime}$ is the Gâteaux derivative of $F$.

We know (see [5], Proposition 5.5) that if (6) holds for any $M>0$, then the functional $F$ is strictly convex. Also, it is evident that if (7) holds, then $F$ is continuously differentiable. Reciprocally, we can prove in a similar way to that given in [6], Lemma 1.1, for the case of the Euclidean spaces, that if the closed unity ball is compact in the strong topology of the space Banach $V, F^{\prime}$ is continuous and $F$ is strictly convex, then the functions $\alpha_{M}(\tau)=\inf _{\|v-u\|=\tau,\|v\|,\|u\| \leq M, v, u \in K}<F^{\prime}(v)-$ $F^{\prime}(u), v-u>$ and $\beta_{M}(\tau)=\sup _{\|v-u\|=\tau,\|v\|,\|u\| \leq M, v, u \in K}\left\|F^{\prime}(v)-F^{\prime}(u)\right\|_{V^{\prime}}$, exist for any $M>0$, and they satisfy (4), (6), and (5), (7), respectively.

It is evident that if (6) and (7) hold, then for any $u, v \in K,\|u\|,\|v\| \leq M$, we have

$$
\alpha_{M}(\|v-u\|) \leq<F^{\prime}(v)-F^{\prime}(u), v-u>\leq \beta_{M}(\|v-u\|)\|v-u\| .
$$

Following the way in [6] (Lemmas 1.1 and 1.2), we can prove that for any $u, v \in K,\|u\|,\|v\| \leq M$, we have

$$
\begin{aligned}
& <F^{\prime}(u), v-u>+\lambda_{M}(\|v-u\|) \leq F(v)-F(u) \leq \\
& <F^{\prime}(u), v-u>+\mu_{M}(\|v-u\|),
\end{aligned}
$$

where

$$
\lambda_{M}(\tau)=\int_{0}^{\tau} \alpha_{M}(\theta) \frac{d \theta}{\theta}, \quad \mu_{M}(\tau)=\int_{0}^{\tau} \beta_{M}(\theta) d \theta
$$


Now, we consider the minimization problem

$$
u \in K: F(u) \leq F(v), \text { for any } v \in K .
$$

It is well known (see [5]) that if $V$ is a reflexive Banach space and $F$ is strictly convex, differentiable, and coercive if $K$ is not bounded, then the above problem has a unique solution, and this is also the unique solution of the problem

$$
u \in K:\left\langle F^{\prime}(u), v-u>\geq 0, \text { for any } v \in K .\right.
$$

¿From (9) we see that, for a given $M>0$ such that the solution $u$ of (12) satisfies $\|u\| \leq M$, we have

$$
\lambda_{M}(\|v-u\|) \leq F(v)-F(u), \text { for any } v \in K,\|v\| \leq M .
$$

The proposed algorithm corresponding to the subspaces $V_{1}, \cdots, V_{m}$ and the convex set $K$ is written as follows

Algorithm 2.1. We start the algorithm with an arbitrary $u^{0} \in K$. At iteration $n+1$, having $u^{n} \in K, n \geq 0$, we compute sequentially for $i=1, \ldots, m, w_{i}^{n+1} \in V_{i}$ satisfying

$$
w_{i}^{n+1}=\arg \min _{u^{n+\frac{i-1}{m}}+v_{i} \in K, v_{i} \in V_{i}} G\left(v_{i}\right)
$$

with $G\left(v_{i}\right)=F\left(u^{n+\frac{i-1}{m}}+v_{i}\right)$, and then we update $u^{n+\frac{i}{m}}=u^{n+\frac{i-1}{m}}+$ $w_{i}^{n+1}$.

This algorithm does not assume a decomposition of the convex set $K$ depending on the subspaces $V_{i}$. As for problem (11), since the subspaces $V_{i}$ are reflexive Banach spaces, problem (14) has a unique solution, $w_{i}^{n+1} \in V_{i}, u^{n+\frac{i-1}{m}}+w_{i}^{n+1} \in K$, and it also satisfies the variational inequality

$$
\begin{aligned}
& <F^{\prime}\left(u^{n+\frac{i-1}{m}}+w_{i}^{n+1}\right), v_{i}-w_{i}^{n+1}>\geq 0, \\
& \text { for any } v_{i} \in V_{i}, u^{n+\frac{i-1}{m}}+v_{i} \in K .
\end{aligned}
$$

We have the following general convergence result.

Theorem 1 We consider that $V$ is a reflexive Banach, $V_{1}, \cdots, V_{m}$ are some closed subspaces of $V, K$ is a non empty closed convex subset of $V$, and $F$ is Gâteaux differentiable functional on $K$ which is assumed to be coercive if $K$ is not bounded. If Assumption 2.1 holds, and for any $M>0$ there exist two functions $\alpha_{M}$ and $\beta_{M}$ satisfying (4)-(7), then, for any $i=1, \cdots, m, u^{n+\frac{i}{m}} \rightarrow u$, strongly in $V$, as $n \rightarrow \infty$, where $u$ is the 
solution of problem (11) and $u^{n+\frac{i}{m}}$ are given by Algorithm 2.1 starting from an arbitrary given $u^{0}$.

Proof. From (15) and (9), we have for any $n \geq 0$ and $i=1, \cdots, m$,

$$
F\left(u^{n+\frac{i-1}{m}}\right)-F\left(u^{n+\frac{i}{m}}\right) \geq \lambda_{M}\left(\left\|w_{i}^{n+1}\right\|\right),
$$

and therefore, using (11), we get for any $n \geq 0$ and $i=1, \cdots, m$, that

$$
F(u) \leq F\left(u^{n+\frac{i}{m}}\right) \leq F\left(u^{n+\frac{i-1}{m}}\right) \leq F\left(u^{0}\right) .
$$

Taking into account the boundedness of $K$ or the coerciveness of $F$, it follows that there exists a real constant $M>0$ such that

$$
\|u\| \leq M,\left\|u^{0}\right\| \leq M,\left\|u^{n+\frac{i}{m}}\right\| \leq M \forall n \geq 0, i=1, \cdots, m .
$$

¿From (16) we also get

$$
F\left(u^{n}\right)-F\left(u^{n+1}\right) \geq \sum_{i=1}^{m} \lambda_{M}\left(\left\|w_{i}^{n+1}\right\|\right), \text { for any } n \geq 0 .
$$

Consequently, from (17), the series $\sum_{n=1}^{\infty} \lambda_{M}\left(\left\|w_{i}^{n+1}\right\|\right)$ is convergent for any $i=1, \cdots, m$, and therefore

$$
\left\|w_{i}^{n+1}\right\| \rightarrow 0, \text { as } n \rightarrow \infty, \text { for any } i=1, \cdots, m .
$$

Applying Assumption 2.1 for $w=u^{n}, v=u$, and $w_{i}=w_{i}^{n+1}$, we get a decomposition $u_{1}, \cdots, u_{m}$ of $u-u^{n}$. From (1), we can replace $v_{i}$ by $u_{i}$ in (15), and we have $\left\langle F^{\prime}\left(u^{n+\frac{i}{m}}\right)-F^{\prime}\left(u^{n+1}\right), u_{i}-w_{i}^{n+1}>\right.$ $+<F^{\prime}\left(u^{n+1}\right), u_{i}-w_{i}^{n+1} \geq 0$. Using (2) we have $\sum_{i=1}^{m}<F^{\prime}\left(u^{n+\frac{i}{m}}\right)-$ $F^{\prime}\left(u^{n+1}\right), u_{i}-w_{i}^{n+1}>+<F^{\prime}\left(u^{n+1}\right), u-u^{n+1}>\geq 0$. Using this inequality, from (18), (9) and (7) we obtain

$$
\begin{aligned}
& F\left(u^{n+1}\right)-F(u)+\lambda_{M}\left(\left\|u-u^{n+1}\right\|\right) \leq<F^{\prime}\left(u^{n+1}\right), u^{n+1}-u> \\
& \leq \sum_{i=1}^{m}<F^{\prime}\left(u^{n+\frac{i}{m}}\right)-F^{\prime}\left(u^{n+1}\right), u_{i}-w_{i}^{n+1}> \\
& =\sum_{i=1}^{m} \sum_{j=i+1}^{m}<F^{\prime}\left(u^{n+\frac{j-1}{m}}\right)-F^{\prime}\left(u^{n+\frac{j}{m}}\right), u_{i}-w_{i}^{n+1}> \\
& \leq \sum_{i=1}^{m} \beta_{M}\left(\left\|w_{i}^{n+1}\right\|\right) \sum_{i=1}^{m}\left\|u_{i}-w_{i}^{n+1}\right\| .
\end{aligned}
$$

¿From (20) and (3) we get that the sequence $\left\{\sum_{i=1}^{m}\left\|u_{i}-w_{i}^{n+1}\right\|\right\}_{n}$ is bounded. Also, from (20) and (5) we have $\sum_{i=1}^{m} \beta_{M}\left(\left\|w_{i}^{n+1}\right\|\right) \rightarrow 0$ as $n \rightarrow \infty$. Consequently, $F\left(u^{n+1}\right)-F(u) \rightarrow 0$ and $\lambda_{M}\left(\left\|u-u^{n+1}\right\|\right) \rightarrow 0$ as $n \rightarrow \infty$. Now, from (4) and (10) it is clear that $u^{n} \rightarrow u$ as $n \rightarrow \infty$. 


\section{Error estimate}

The error estimate essentially stands on the convergence order of the functions $\alpha_{M}(\tau)$ and $\beta_{M}(\tau)$ to zero as $\tau \rightarrow 0$. In the following we take these functions of polynomial form

$$
\alpha_{M}(\tau)=A_{M} \tau^{p}, \quad \beta_{M}(\tau)=B_{M} \tau^{q-1},
$$

where $A_{M}>0, B_{M}>0, p>1$ and $q>1$ are some real constants. We have marked here that the constants $A_{M}$ and $B_{M}$ depend on $M$, and we see from (8) that we must take $p \geq q$. Now, from (10) we get

$$
\lambda(\tau)=\frac{A_{M}}{p} \tau^{p}, \quad \mu(\tau)=\frac{B_{M}}{q} \tau^{q} .
$$

Naturally, the convergence rate will depend on the spaces $V_{1}, \cdots, V_{m}$, and we shall consider the following form of Assumption 2.1 having condition (3) slightly modified

Assumption 3.1. There exists a constant $C_{0}$ such that for any $w, v \in K$ and $w_{i} \in V_{i}$ with $w+\sum_{j=1}^{i} w_{j} \in K, i=1, \cdots, m$, there exist $v_{i} \in V_{i}$, $i=1, \cdots, m$, satisfying (1), (2) and

$$
\sum_{i=1}^{m}\left\|v_{i}\right\|^{p} \leq C_{0}^{p}\left(\|v-w\|^{p}+\sum_{i=1}^{m}\left\|w_{i}\right\|^{p}\right) .
$$

In the case of the minimization of quadratic functionals in [3], the above assumption has been introduced for $p=2$. The following theorem is a generalization for nonlinear inequalities of the result in [9] concerning the convergence of the method for nonlinear equations.

Theorem 2 On the conditions of Theorem 1 we consider the functions $\alpha_{M}$ and $\beta_{M}$ defined in (22) and we make Assumption 3.1. If $u$ is the solution of problem (11) and $u^{n}, n \geq 0$, are its approximations obtained from Algorithm 2.1, then we have the following error estimations:

(i) if $p=q$ we have

$$
\begin{aligned}
& F\left(u^{n}\right)-F(u) \leq\left(\frac{\hat{C}}{\hat{C}+1}\right)^{n}\left[F\left(u^{0}\right)-F(u)\right] \\
& \left\|u^{n}-u\right\|^{p} \leq \frac{\hat{C}+1}{\bar{C}}\left(\frac{\hat{C}}{\hat{C}+1}\right)^{n}\left[F\left(u^{0}\right)-F(u)\right] .
\end{aligned}
$$

(ii) if $p>q$ we have

$$
\begin{aligned}
& F\left(u^{n}\right)-F(u) \leq \frac{F\left(u^{0}\right)-F(u)}{\left[1+n \tilde{C}\left(F\left(u^{0}\right)-F(u)\right)^{\frac{p-q}{q-1}}\right]^{\frac{q-1}{p-q}}}, \\
& \left\|u-u^{n}\right\|^{p} \leq \frac{\hat{C}}{\bar{C}} \frac{\left(F\left(u^{0}\right)-F(u)\right)^{\frac{q-1}{p-1}}}{\left[1+(n-1) \tilde{C}\left(F\left(u^{0}\right)-F(u)\right)^{\frac{p-q}{q-1}}\right]^{\frac{(q-1)^{2}}{(p-1)(p-q)}}} .
\end{aligned}
$$


The constants $\hat{C}, \bar{C}$ and $\tilde{C}$ are given in (28), (31) and (33), respectively.

Proof. From (21), using $\lambda_{M}$ in (23), $\beta_{M}$ in (22), and (24) in which we take $v_{i}=u_{i}, v=u, w=u^{n}$ and $w_{i}=w_{i}^{n+1}$, we have $F\left(u^{n+1}\right)-$ $F(u)+\frac{A_{M}}{p}\left\|u-u^{n+1}\right\|^{p} \leq B_{M} \sum_{i=1}^{m}\left\|w_{j}^{n+1}\right\|^{q-1} \sum_{i=1}^{m}\left\|u_{i}-w_{i}^{n+1}\right\| \leq$ $B_{M} m^{2-\frac{q}{p}}\left(\sum_{i=1}^{m}\left\|w_{i}^{n+1}\right\|^{p}\right)^{\frac{q-1}{p}}\left[\left(\sum_{i=1}^{m}\left\|w_{i}^{n+1}\right\|^{p}\right)^{\frac{1}{p}}+\left(\sum_{i=1}^{m}\left\|u_{i}\right\|^{p}\right)^{\frac{1}{p}}\right] \leq B_{M}$ $m^{2-\frac{q}{p}}\left(\sum_{i=1}^{m}\left\|w_{i}^{n+1}\right\|^{p}\right)^{\frac{q-1}{p}}\left[\left(1+C_{0}\right)\left(\sum_{i=1}^{m}\left\|w_{i}^{n+1}\right\|^{p}\right)^{\frac{1}{p}}+C_{0}\left\|u-u^{n}\right\|\right]$. Therefore, using (13) with $v=u^{n}$, (19), and $\lambda_{M}$ given in (23), we have $F\left(u^{n+1}\right)-F(u)+\frac{A_{M}}{p}\left\|u-u^{n+1}\right\|^{p} \leq B_{M}\left(\frac{p}{A_{M}}\right)^{\frac{q}{p}} m^{2-\frac{q}{p}}\left(F\left(u^{n}\right)-F\left(u^{n+1}\right)\right)^{\frac{q-1}{p}}$ $\left[\left(1+C_{0}\right)\left(F\left(u^{n}\right)-F\left(u^{n+1}\right)\right)^{\frac{1}{p}}+C_{0}\left(F\left(u^{n}\right)-F(u)\right)^{\frac{1}{p}}\right] \leq B_{M}\left(\frac{p}{A_{M}}\right)^{\frac{q}{p}} m^{2-\frac{q}{p}}$ $\left(F\left(u^{n}\right)-F\left(u^{n+1}\right)\right)^{\frac{q-1}{p}}\left[\left(1+2 C_{0}\right)\left(F\left(u^{n}\right)-F\left(u^{n+1}\right)\right)^{\frac{1}{p}}+C_{0}\left(F\left(u^{n+1}\right)-\right.\right.$ $\left.F(u)^{\frac{1}{p}}\right]$. But, for some given $\eta>0$ and $\zeta>0$, we have $\zeta x^{\frac{1}{p}}-\eta x \leq$ $\left(\frac{\zeta^{p}}{\eta}\right)^{\frac{1}{p-1}}$, for any $x \geq 0$. Consequently, for a $0<\eta<1$, subtracting $\eta\left(F\left(u^{n+1}\right)-F(u)\right)$ from both sides of the last inequality, we get

$$
\begin{aligned}
& F\left(u^{n+1}\right)-F(u)+\frac{A_{M}}{p(1-\eta)}\left\|u-u^{n+1}\right\|^{p} \leq \\
& \hat{C}\left[F\left(u^{n}\right)-F\left(u^{n+1}\right)\right]^{\frac{q-1}{p-1}},
\end{aligned}
$$

where

$$
\begin{aligned}
& \hat{C}=B_{M}\left(\frac{p}{A_{M}}\right)^{\frac{q}{p}} m^{2-\frac{q}{p}}\left[\left(1+2 C_{0}\right)\left(F\left(u^{0}\right)-F(u)\right)^{\frac{p-q}{p(p-1)}}+\right. \\
& \left.\left(B_{M}\left(\frac{p}{A_{M}}\right)^{\frac{q}{p}} m^{2-\frac{q}{p}}\right)^{\frac{1}{p-1}} C_{0}^{\frac{p}{p-1}} / \eta^{\frac{1}{p-1}}\right] /(1-\eta),
\end{aligned}
$$

and we have used (17) to write $F\left(u^{n}\right)-F\left(u^{n+1}\right) \leq F\left(u^{0}\right)-F(u)$. From (27) we have

$$
\left[F\left(u^{n+1}\right)-F(u)\right] \leq \hat{C}\left[F\left(u^{n}\right)-F\left(u^{n+1}\right)\right]^{\frac{q-1}{p-1}} .
$$

Using again (17) we have $F\left(u^{n}\right)-F\left(u^{n+1}\right) \leq F\left(u^{n}\right)-F(u)$, and from (13) and (23) we get $\frac{A_{M}}{p}\left\|u^{n+1}-u\right\|^{p} \leq F\left(u^{n+1}\right)-F(u)$. From these two last inequalities and (27) we get

$$
\left\|u-u^{n+1}\right\|^{p} \leq \frac{\hat{C}}{\bar{C}}\left[F\left(u^{n}\right)-F(u)\right]^{\frac{q-1}{p-1}},
$$

where

$$
\bar{C}=\frac{(2-\eta) A_{M}}{(1-\eta) p} .
$$

Now, if $p=q$, we can easily find (25) from (29) and (30). If $p \neq q$, we get from (29) that $F\left(u^{n+1}\right)-F(u)+\frac{1}{\hat{C}^{\frac{p-1}{q-1}}}\left[F\left(u^{n+1}\right)-F(u)\right]^{\frac{p-1}{q-1}} \leq$ 
$F\left(u^{n}\right)-F(u)$, and applying Lemma 3.2 in [9] we get $F\left(u^{n+1}\right)-F(u) \leq$ $\left[\tilde{C}+\left(F\left(u^{n}\right)-F(u)\right)^{\frac{q-p}{q-1}}\right]^{\frac{q-1}{q-p}}$, or

$$
F\left(u^{n+1}\right)-F(u) \leq\left[(n+1) \tilde{C}+\left(F\left(u^{0}\right)-F(u)\right)^{\frac{q-p}{q-1}}\right]^{\frac{q-1}{q-p}}
$$

where

$$
\tilde{C}=\frac{p-q}{(p-1)\left(F\left(u^{0}\right)-F(u)\right)^{\frac{p-q}{q-1}}+(q-1) \hat{C}^{\frac{p-1}{q-1}}} .
$$

Equation (32) is another form of the first estimate in (26), and the second one can be obtained using (32) and (30). The value of $\eta$ in the the expression of $\hat{C}$ and $\bar{C}$ can be arbitrary in $(0,1)$. On the other hand, we see that the constants in the error estimations of $F\left(u^{n}\right)-F(u)$ in (25) and (26) are some increasing functions of $\hat{C}$, and there is an $\eta_{0} \in(0,1)$ such that $\hat{C}\left(\eta_{0}\right) \leq \hat{C}(\eta)$ for any $\eta \in(0,1)$. This value $\eta_{0}$ can be found by solving a nonlinear algebraic equation.

\section{The multiplicative Schwarz method as a subspace correction method}

We shall prove in the following that for the problems in which we seek for the solution in a Sobolev space, Assumption 3.1 holds, and consequently, the convergence and error estimation theorems hold, too.

Let $\Omega$ be an open bounded domain in $\mathbf{R}^{d}$ with Lipschitz continuous boundary $\partial \Omega$. We take $V=W_{0}^{1, s}(\Omega), 1<s<\infty$, and a convex closed set $K \subset V$ satisfying

Property 4.1. If $v, w \in K$, and if $\theta \in C^{1}(\Omega)$ with $0 \leq \theta \leq 1$, then $\theta v+(1-\theta) w \in K$.

We consider an overlapping decomposition of the domain $\Omega$,

$$
\Omega=\bigcup_{i=1}^{m} \Omega_{i}
$$

in which $\Omega_{i}$ are open subdomains with Lipschitz continuous boundary. We associate to the domain decomposition (34) the subspaces $V_{i}=W_{0}^{1, s}\left(\Omega_{i}\right), i=1, \cdots, m$. In this case, Algorithm 2.1 represents a multiplicative Schwarz method.

Remark 4.1 The above spaces $V$ and $V_{i}$ correspond to Dirichlet boundary conditions. Similar results can be obtained if we consider mixed boundary conditions. We take $\partial \Omega=\bar{\Gamma}_{1} \cup \bar{\Gamma}_{2}, \Gamma_{1} \cap \Gamma_{2}=\emptyset$ a partition of 
the boundary such that meas $\left(\Gamma_{1}\right)>0$, and we consider the Sobolev space $V=\left\{v \in W^{1, s}(\Omega): v=0\right.$ on $\left.\Gamma_{1}\right\}$. The subspaces $V_{i}$ will be defined in this case as $V_{i}=\left\{v_{i} \in W^{1, s}(\Omega): v_{i}=0\right.$ in $\Omega-\bar{\Omega}_{i}, v_{i}=0$ in $\left.\partial \Omega_{i} \cap \Gamma_{1}\right\}$, $i=1, \cdots, m$.

Also, we have considered problems having the solution in $W^{1, s}(\Omega)$, but all the obtained results hold with $\left[W^{1, s}(\Omega)\right]^{N}, N \geq 2$, in the place of $W^{1, s}(\Omega)$.

Concerning the decomposition (34), we assume that there are some functions $\theta_{j}^{i} \in C^{1}(\bar{\Omega}), i=1, \cdots, m, j=i, \cdots, m$ such that for any $i=1, \cdots, m$ we have

$$
\operatorname{supp}\left(\theta_{j}^{i}\right) \subset\left(\bar{\Omega}_{j}\right), 0 \leq \theta_{j}^{i} \leq 1, j=i, \cdots, m, \sum_{j=i}^{m} \theta_{j}^{i} \equiv 1 \text { in } \bigcup_{j=i}^{m} \Omega_{j} .
$$

This is a easy enough constraint on the domain decomposition (34). In [8] or [1], for instance, some conditions in which a domain decomposition satisfies (35) are given.

Proposition 4.1 If the domain decomposition (34) satisfies (35), then Assumption 3.1 holds for any convex set $K$ having Property 4.1 .

Proof. The proof is similar to that given in [2] and we only outline it. Let us consider $w \in K, w_{i} \in V_{i}$ such that $w+\sum_{j=1}^{i} w_{j} \in K$, $i=1, \cdots, m$, and let $v$ be another element in $K$. First we define $v_{1}=$ $\theta_{1}^{1}(v-w)+\left(1-\theta_{1}^{1}\right) w_{1}$ and we prove that: $v_{1} \in V_{1}, w+v_{1} \in K$, $v-v_{1}+w_{1} \in K, v-w-v_{1} \in W_{0}^{1, s}\left(\bigcup_{i=2}^{m} \Omega_{i}\right)$, and $v-w-v_{1}=0$ in $\Omega \backslash \overline{\cup_{i=2}^{m} \Omega_{i}}$. For $2 \leq i \leq m-1$, taking $v_{i}=\theta_{i}^{i}\left(v-w-\sum_{j=1}^{i} v_{j}\right)+\left(1-\theta_{i}^{i}\right) w_{i}$ we recursively prove that: $v_{i} \in V_{i}, v_{i}+w+\sum_{j=1}^{i-1} w_{j} \in K, v-\sum_{j=1}^{i} v_{j}+$ $\sum_{j=1}^{i} w_{j} \in K, v-w-\sum_{j=1}^{i} v_{j} \in W_{0}^{1, s}\left(\bigcup_{j=i+1}^{m} \Omega_{j}\right)$, and $v-w-\sum_{j=1}^{i} v_{j}=0$ in $\Omega-\overline{\cup_{j=i+1}^{m} \Omega_{j}}$. Finally, defining $v_{m}=v-w-\sum_{j=1}^{m-1} v_{j}$, we get that (1) and (2) hold. Also, (24) in Assumption 3.1 holds, in which $C_{0}$ depends on the unity partitions (35), but it is independent of $w, v, w_{i}$ and $v_{i}$.

\section{Numerical example}

For a domain $\Omega \subset \mathbf{R}^{d}$ and an $1<s<\infty$, let $K \subset V \equiv W_{0}^{1, s}(\Omega)$ be a closed and convex set. Given an $f \in V^{\prime} \equiv W^{-1, s^{\prime}}(\Omega), 1 / s+1 / s^{\prime}=1$, we consider the problem

$$
u \in K: \int_{\Omega}|\nabla u|^{s-2} \nabla u \nabla(v-u) \geq f(v-u), \text { for any } v \in K .
$$


The solution $u \in K$ of the above inequality is also the solution of the minimization problem $F(u)=\min _{v \in K} F(v)$, where $F(v)=\frac{1}{s} \int_{\Omega}|\nabla v|^{s}-$ $f(v)$. We know (see [7]) that if $1<s \leq 2$, then there exist two positive constants $\alpha$ and $\beta$ such that $\left\langle F^{\prime}(v)-F^{\prime}(u), v-u>\geq \alpha \frac{\|v-u\|_{1, s}^{2}}{\left(\|v\|_{1, s}+\|u\|_{1, s}\right)^{2-s}}\right.$, $\beta\|v-u\|_{1, s}^{s-1} \geq\left\|F^{\prime}(v)-F^{\prime}(u)\right\|_{V^{\prime}}$, for any $v, u \in W_{0}^{1, s}(\Omega)$. Consequently, the functions introduced in (22) can be written as $\alpha_{M}(\tau)=\frac{\alpha}{(2 M)^{2-s}} \tau^{2}$, $\beta_{M}(\tau)=\beta \tau^{s-1}$, and therefore, $A_{M}=\frac{\alpha}{(2 M)^{2-s}}, B_{M}=\beta, p=2$ and $q=s$ in (22). If $s \geq 2$, then there exist two positive constants $\alpha$ and $\beta$ such that (see [4]) $<F^{\prime}(v)-F^{\prime}(u), v-u>\geq \alpha\|v-u\|_{1, s}^{s}$, $\beta\left(\|v\|_{1, s}+\|u\|_{1, s}\right)^{s-2}\|v-u\|_{1, s} \geq\left\|F^{\prime}(v)-F^{\prime}(u)\right\|_{V^{\prime}}$, for any $v, u \in$ $W_{0}^{1, s}(\Omega)$. Therefore, for a given $M>0$, we have $\alpha_{M}(\tau)=\alpha \tau^{s}, \beta_{M}(\tau)=$ $\beta(2 M)^{s-2} \tau$, and therefore, $A_{M}=\alpha, B_{M}=\beta(2 M)^{s-2}, p=s$ and $q=2$ in (22). We can conclude from the above comments that Algorithm 2.1 can be applied for the solving of problem (36) if the convex set $K$ has Property 4.1. Naturally, the error estimation in Section 3 hold.

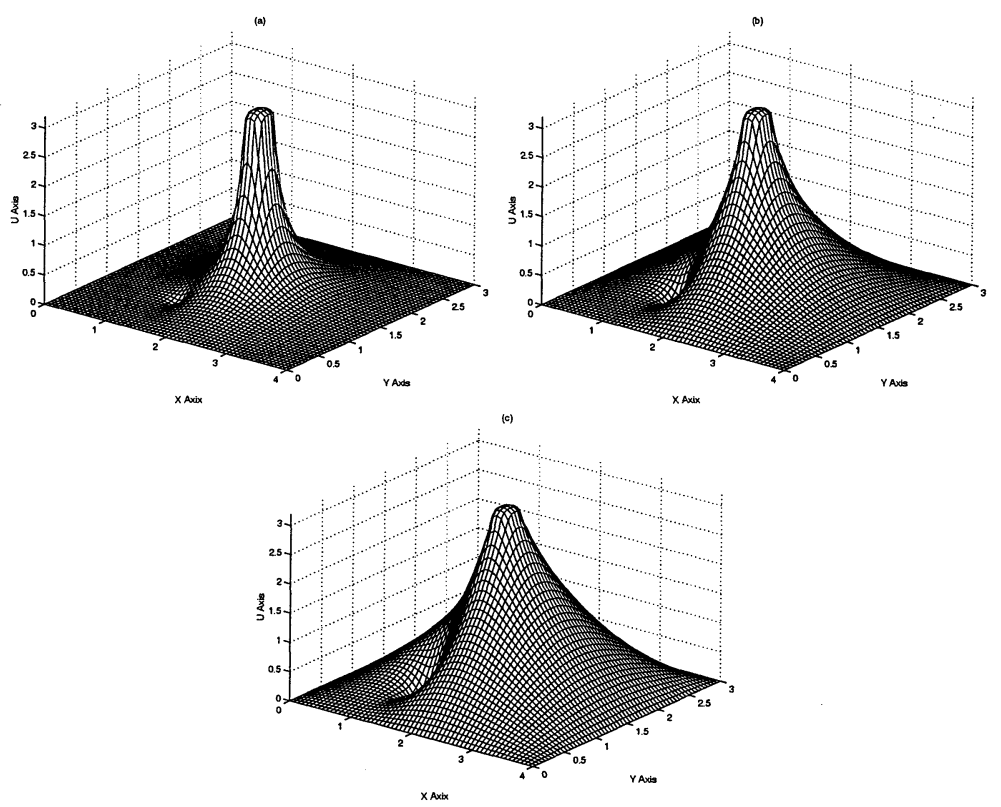

Figure 5.1. Solution for: (a) $\mathrm{s}=1.5$, (b) $\mathrm{s}=2$., (c) $\mathrm{s}=3$.

If $\Omega \subset \mathbf{R}^{2}$ and the convex set is of the form $K=[a, b]$, where $a, b \in$ $W_{0}^{1, s}(\Omega), a \leq b$, then (36) is the problem of a nonlinear elastic membrane stretched over the obstacle $a$ and under obstacle $b$. We have plotted in Figure 5.1 three computed solutions, corresponding to $s=1.5, s=2.0$ and $s=3.0$, of such a problem having a rectangular domain. In this example, the exterior forces $f$ are zero. In a subsequent paper we shall 
present an analysis of the one and two-level Schwarz method in the finite element spaces, where the introduced assumption hold, too. In these cases, we are able to explicitely write the constant $C_{0}$ introduced in Assumption 3.1, as well as the constants in the error estimations of Theorem 2, as a function of the mesh and domain decomposition parameters.

\section{Acknowledgments}

The author acknowledges the financial support of IMAR under the contract nr. ICA1-CT-2000-70022 with the European Commission for this study.

\section{References}

[1] L. Badea, A generalization of the Schwarz alternating method to an arbitrary number of subdomains, Numer. Math., 55 (1989), pp. 61-81.

[2] L. Badea, On the Schwarz alternating method with more than two subdomains for nonlinear monotone problems, SIAM J. Numer. Anal., 28 (1991), pp. 179204.

[3] L. Badea, X.-C. Tai and J. Wang, Convergence rate analysis of a multiplicative Schwarz method for variational inequalities, SIAM J. Numer. Anal., submitted, 2001.

[4] Philippe G. Ciarelet, The Finite Element Method for Elliptic Problems, NorthHolland, Amsterdam, 1978.

[5] I. Ekeland and R. Temam, Convex analysis and variational problems, NorthHolland, Amsterdam, 1976.

[6] R. Glowinski, J. L. Lions and R. Trémolières, Analyse numérique des inéquations variationnelles, Dunod, 1976.

[7] R. Glowinski and A. Marrocco, Sur l'aproximation par éléments finis d'ordre un, et la résolution par pénalisation-dualité, d'une classe de problèmes de Dirichlet non linéaires, Rev. Francaise Automat. Informat. Recherche Opérationnelle, Sér. Rouge Anal. Numér., R-2, 1975, pp. 41-76.

[8] P. L. Lions, On the Schwarz alternating method I, in R. Glowinski et al., eds., First International Symposium on Domain Decomposition Methods for Partial Differential Equations, Philadelphia, SIAM, 1988, pp. 2-42.

[9] X.-C. Tai and J. Xu, Global and uniform convergence of subspace correction methods for some convex optimization problems, Math. of Comp., electronically published on 11 May, 2001. 